Pacific Journal of Mathematics

VOLTERRA TRANSFORMATIONS OF THE WIENER
MEASURE ON THE SPACE OF CONTINUOUS FUNCTIONS OF
TWO VARIABLES

TWO VARIABLES 


\title{
VOLTERRA TRANSFORMATIONS OF THE WIENER MEASURE ON THE SPACE OF CONTINUOUS FUNCTIONS OF TWO VARIABLES
}

\author{
WILLIAM N. HUDSON
}

The transformation of Wiener integrals over the space $C_{2}$ of continuous functions of two variables by a Volterra operator $T$ is investigated. The operator $T$ is defined for functions $x \in C_{2}$ by

$$
T x(s, t)=x(s, t)+\int_{0}^{s} \int_{0}^{t} K(u, v) x(u, v) d u d v,
$$

where the kernel $K(u, v)$ is continuous. A stochastic integral analogous to $K$. Ito's is defined and used to determine a Jacobian $J(x)$ for $T$ such that if $F(x)$ is a Wiener measurable functional, $\Gamma$ a Wiener measurable set, and $m$ Wiener measure, $\int_{\Gamma} F(x) d m=\int_{T^{-1}(\Gamma)} F(T x) J(x) d m$.

Let $C_{2}$ be the collection of real valued functions $f$ defined on $D=$ $[0,1] \times[0,1]$ such that $f(0, t)=f(s, 0)=0$. The space $C_{2}$ is topologized by the sup-norm. In [3], Yeh defined a measure $m$ on $C_{2}$ over the Borel $\sigma$-algebra and extended it to the Caratheodory $\sigma$-algebra relative to $m$. It is the purpose of this paper to investigate the transformation of the measure $m$ when the elements of $C_{2}$ are transformed by a Volterra integral operator of the second kind. The effect of such transformations in the Wiener space of continuous functions of one variable was studied by Cameron and Martin in [1].

Let $0=s_{0}<s_{1}<\cdots<s_{m} \leqq 1$ and $0=t_{0}<t_{1}<\cdots<t_{n} \leqq 1$ and let $E$ be a $n m$-dimensional Borel set. We denote by $\mathfrak{F}\left(s_{1}, \cdots, s_{m}, t_{1}, \cdots, t_{n}\right)$ the $\sigma$-algebra of sets of the form $\left\{x \in C_{2}:\left[x\left(s_{1}, t_{1}\right), \cdots, x\left(s_{m}, t_{n}\right)\right] \in E\right\}$ and let $\mathfrak{F}_{0}=\cup \mathfrak{F}\left(s_{1}, \cdots, s_{m}, t_{1}, \cdots, t_{n}\right)$ where the union is over all such partitions of $D$. The measure $m$ is given on $\mathfrak{F}\left(s_{1}, \cdots, s_{m}, t_{1}, \cdots, t_{n}\right)$ by

$$
\begin{aligned}
& m\left\{x \in C_{2}:\left[x\left(s_{1}, t_{1}\right), \cdots, x\left(s_{m}, t_{n}\right)\right] \in E\right\} \\
& =K\left(s_{1}, \cdots, s_{m}, t_{1}, \cdots, t_{n}\right) \\
& \quad \cdot \int(m n) \int W\left(s_{1}, \cdots, s_{m}, t_{1}, \cdots, t_{n}, u_{11}, \cdots, u_{m n}\right) d u_{11}, \cdots, d u_{m n},
\end{aligned}
$$

where

$$
\begin{aligned}
& K\left(s_{1}, \cdots, s_{m}, t_{1}, \cdots, t_{n}\right) \\
& \quad=\left\{(2 \pi)^{-m n}\left[s_{1}\left(s_{2}-s_{1}\right) \cdots\left(s_{m}-s_{m-1}\right)\right]^{n}\left[t_{1}\left(t_{2}-t_{1}\right) \cdots\left(t_{n}-t_{n-1}\right)\right]^{m}\right\}^{\frac{1}{2}},
\end{aligned}
$$




$$
\begin{aligned}
& W\left(s_{1}, \cdots, s_{m}, t_{1}, \cdots, t_{n}, u_{11}, \cdots, u_{m n}\right) \\
& \quad=\exp \left\{-\frac{1}{2} \sum_{i=1}^{m} \sum_{j=1}^{n} \frac{\left(u_{i j}-u_{i-1, j}-u_{i, j-1}+u_{i-1, j-1}\right)^{2}}{\left(s_{i}-s_{i-1}\right)\left(t_{j}-t_{j-1}\right)}\right\},
\end{aligned}
$$

and $u_{0, i}=u_{i, 0}=0$. Yeh showed that $m$ was a probability measure over $\left(C_{2}, \mathfrak{F}_{0}\right)$ and considered the Caratheodory extension $\widetilde{F}$ of the algebra $\widetilde{\mho}_{0}$ relative to $m$. It is well known that $\widetilde{F}$ contains the Borel $\sigma$-algebra.

We consider the stochastic process $X(s, t, x)=x(s, t), x \in C_{2} . \quad X(s, t)$ is analogous to ordinary Brownian motion and proceeding accordingly, we define a stochastic integral analogous to Ito's and denote such integrals of a process $f(s, t, x)$ on $C_{2}$ by $\int_{D} f(s, t, x) d X$.

Next, the Volterra operator $T$ defined by

$$
(T: x)(s, t)=x(s, t)+\int_{0}^{s} \int_{0}^{t} K(u, v) x(u, v) d u d v
$$

is considered. The kernel $K(s, t)$ of $T$ is assumed to be continuous over the unit square $D$. It is well-known that $T$ is a one-to-one map of $C_{2}$ onto $C_{2}$ with a bounded inverse. We can now state our main results.

\section{Statement of main results.}

THEOREM 1. Let $F(x)$ be bounded and continuous on $C_{2}$ and vanish outside a bounded subset of $C_{2}$. Let $K(s, t)$ be continuous over the unit square $D$. Then

$$
\int_{C_{2}} F(x) d m(x)=\int_{C_{2}} F(T x) J(x) d m(x)
$$

where $(T x)(s, t)=x(s, t)+\int_{0}^{s} \int_{0}^{t} K(u, v) x(u, v) d u d v, x \in C_{2}$ and $J(x)$ is given by the formula

$$
J(x)=\exp \left\{-\int_{D} K(u, v) X(u, v) d X-\frac{1}{2} \int_{D} K(u, v)^{2} x(\imath, v)^{2} d u d v\right\} \cdot
$$

The first integral in the expression for $J(x)$ is the stochastic integral of the process $K(s, t) X(s, t, x)$ with respect to the process $X(s, t, x)=$ $x(s, t)$.

Theorem 2. Let $T$ and $J(x)$ be as in Theorem 1. Then for every $\Gamma \in \mathfrak{\mho}, T^{-1}(\Gamma) \in \mathfrak{F}$ and $T(\Gamma) \in \mathfrak{F}$ and

$$
m(\Gamma)=\int_{T^{-1}(\Gamma)} J(x) d m(x) \quad \text { and }
$$




$$
m(T(\Gamma))=\int_{\Gamma} J(x) d m(x) .
$$

Furthermore, if $F(x), x \in C_{2}$ is measurable with respect to $\mathfrak{F}$, then

$$
\begin{gathered}
\int_{\Gamma} F(x) d m(x)=\int_{T^{-1}(\Gamma)} F(T x) J(x) d m(x) \\
\int_{T(\Gamma)} F(x) d m(x)=\int_{\Gamma} F(T x) J(x) d m(x)
\end{gathered}
$$

in the sense that the existence of one side implies the existence of the other and the equality of the two.

3. Definition of the stochastic integral. In this section the basic definition of the stochastic integral is given and some fundamental properties are listed. The proofs are omitted since they are strictly analogous to those of $K$. Itô in [2].

Let $(\Omega, \mathfrak{B}, P)$ be a probability space and let $\{X(s, t): s, t \in[0,1]\}$ be a stochastic process with two time parameters defined over $(\Omega, \mathfrak{B}, P)$. If for any pair $(m, n)$ of positive integers, and any set $S=\left\{a_{1}, \cdots a_{m}\right.$, $\left.b_{1}, \cdots, b_{m}, c_{1}, \cdots, c_{n}, d_{1}, \cdots, d_{n}\right\}$ of real numbers in $[0,1]$ such that $a_{1}<b_{1} \leqq a_{2}<b_{2} \leqq \cdots \leqq a_{m}<b_{m}$ and $c_{1}<d_{1} \leqq c_{2}<d_{2} \leqq \cdots \leqq c_{n}<d_{n}$, the "increments" $X\left(b_{i}, d_{j}\right)-X\left(a_{i}, d_{j}\right)-X\left(b_{i}, c_{j}\right)+X\left(a_{i}, c_{j}\right) i=1, \cdots, m$, $j=1, \cdots, n$ are independent random variables, the process $X(s, t)$ will be called biadditive. If a biadditive process $X(s, t)$ is Gaussian and has the additional properties that for all $(s, t) \in D E(X(s, t))=0$, $\operatorname{var}(X(s, t))=s t$, and $X(0, t)=X(s, 0)=0$, then $X(s, t)$ will be said to be a generalized Brownian motion. The process $X(s, t, x)=x(s, t)$, $x \in C_{2}$ defined on $\left(C_{2}, \mathfrak{F}, m\right)$ is an example of a generalized Brownian motion.

Now let $X(s, t)$ be a fixed generalized Brownian motion and denote the increments $X(b, d)-X(a, d)-X(b, c)+X(a, c)$ by $\Delta(a, c, b, d)$. Let $(\mathcal{D}$ denote the Borel subsets of $D$. For each choice of $(s, t) \in D$, let $\mathfrak{A}(s, t)$ be a sub $\sigma$-algebra of $\mathfrak{B}$ which contains $\sigma\{X(u, v): u \leqq s$ and $v \leqq t\}$, the $\sigma$-algebra generated by $X(s, t)$ up to $(s, t)$, and which is independent of $\sigma\{\Delta(s, t, u, v): u \geqq s$ or $t \geqq v\}$. Assume also that if $s \leqq s^{\prime}$ and $t \leqq t^{\prime}, \mathfrak{u}(s, t) \subset \mathfrak{H}\left(s^{\prime}, t^{\prime}\right)$. Let $\mathfrak{M}$ denote the class of stochastic processes $f(s, t, \omega)$ defined on $(\Omega, \mathfrak{B}, P)$ with domain of definition $D$ which satisfy

(i ) $f(s, t, \omega)$ is $\mathfrak{D} \times \mathfrak{B}$ measurable and

(ii) $f(s, t, \cdot)$ is $\mathfrak{u}(s, t)$ measurable.

$\mathfrak{M}_{0}$ will denote the subset of $\mathfrak{M}$ such that if $f(s, t, \omega) \in \mathfrak{M}_{0}$, there are real numbers $0=\alpha_{0}<\alpha_{1}<\cdots<\alpha_{m}=1$ and $0=\beta_{0}<\beta_{1}<\cdots<\beta_{n}=1$ such that $f(s, t)=f\left(\alpha_{j-1}, \beta_{k-1}\right)$ whenever $\alpha_{j-1} \leqq s<\alpha_{j}$ and $\beta_{k-1} \leqq t<\beta_{k}$. $\mathfrak{M}_{1}$ will denote the subset of $\mathfrak{M}$ such that $f(s, t, \omega) \in \mathfrak{M}_{1}$ whenever 
$\int_{D \times \Omega}(f(u, v, \omega))^{2} d P \times \iota<\infty$ where $\measuredangle$ denotes two dimensional Lebesgue measure. $\mathfrak{M}_{2}$ will denote the set of all $f \in \mathfrak{M}$ such that for almost all $\omega \in \Omega, \int_{D} f(u, v, \omega)^{2} d_{\ell}<\infty$. We define the stochastic integral successively for $f \in \mathfrak{M}_{0}$, then for $f \in \mathfrak{M}_{1}$, and finally for $f \in \mathfrak{M}_{2}$.

Definition. For $f(s, t) \in \mathfrak{M}_{0}$, the stochastic integral of $f(s, t)$ with respect to $X(s, t)$ is denoted by $\left(\int f d X\right)(s, t)$ and is defined by

$$
\begin{aligned}
\left(\int f d X\right)(s, t)= & \sum_{p=1}^{j-1} \sum_{q=1}^{k-1} f\left(\alpha_{p-1}, \beta_{q-1}\right) \Delta\left(\alpha_{p-1}, \beta_{q-1}, \alpha_{p}, \beta_{q}\right) \\
& +\sum_{p=1}^{j-1} f\left(\alpha_{p-1}, \beta_{k-1}\right) \Delta\left(\alpha_{p-1}, \beta_{k-1}, \alpha_{p}, t\right) \\
& +\sum_{q=1}^{k-1} f\left(\alpha_{j-1}, \beta_{q-1}\right) \Delta\left(\alpha_{j-1}, \beta_{q-1}, s, \beta_{q}\right) \\
& +f\left(\alpha_{j-1}, \beta_{k-1}\right) \Delta\left(\alpha_{j-1}, \beta_{k-1}, s, t\right) .
\end{aligned}
$$

where the $\alpha$ 's and $\beta$ 's are taken as in the definition of $\mathfrak{M}_{0}$.

The following properties follow from this definition for $f \in \mathfrak{M}_{0}$ in the same way as for the usual stochastic integral.

THEOREM 3.1. If $f \in \mathfrak{M}_{0}$, then $\left(\int f d X\right)(s, t)$ has the following properties.

(1) For $f, g \in \mathfrak{M}_{0}, \omega_{0} \in \Omega$, if $f\left(s, t, \omega_{0}\right)=g\left(s, t, \omega_{0}\right)$ on $D$, then $\left(\int f d X\right)\left(s, t, \omega_{0}\right)=\left(\int g d X\right)\left(s, t, \omega_{0}\right)$ on $D$.

(2) For $f, g \in \mathfrak{M}_{0}$, $\alpha, \beta$ real numbers, $(s, t) \in D\left(\int(\alpha f+\beta g) d X\right)$ $(s, t)=\alpha\left(\int f d X\right)(s, t)+\beta\left(\int g d X\right)(s, t)$.

(3) For almost every $\omega,\left(\int f d X\right)(s, t, \omega)$ is continuous over $D$.

(4) $E\left[\left(\int f d X\right)(s, t)\right]=0$ for all $(s, t) \in D$ and

(5) $\operatorname{var}\left[\left(\int f d X\right)(s, t)\right]=\|f\|_{s, t}^{2}$ where $\|f\|_{s, t}^{2}$

$$
\equiv \int_{[0, s] \times[0, t] \times \Omega} f\left(s^{\prime}, t^{\prime}, \omega\right)^{2} d \iota \times P .
$$

As in the one variable case, $\mathfrak{M}_{0} \cap \mathfrak{M}_{1}$ is dense in $\mathfrak{M}_{1}$ with respect to the Hilbert norm on $L_{2}(D \times \Omega)$. Denoting this norm by $\|$ and using property (5) above, we make the following definition for $f \in \mathfrak{M}_{1}$.

Definition. For $f \in \mathfrak{M}_{1}$, the stochastic integral of $f$ is defined to be 


$$
\left(\int f d X\right)(s, t)=\lim _{n \rightarrow \infty}\left(\int f_{n} d X\right)(s, t)
$$

where the limit on the right is that of convergence in the norm of $L_{2}(\Omega)$ and $\left\{f_{n}\right\}$ is any sequence of functions in $\mathfrak{M}_{0} \cap \mathfrak{M}_{1}$ such that $\lim _{n \rightarrow \infty}\left\|f_{n}-f\right\|=0$.

The following properties hold for functions in $\mathfrak{M}_{1}$.

THEOREM 3.2. Let $f$ and $g$ be in $\mathfrak{M}_{1}$. The following statements are true.

(1) If $f(s, t, \omega)=g(s, t, \omega)$ on $D$ for $\omega \in A \in \mathfrak{B}$, then $\left(\int f d X\right)(s, t, \omega)=$ $\left(\int g d X\right)(s, t, \omega)$ for $\omega \in A_{0} \subset A$ where $A_{0} \in \mathfrak{B}$ and $P\left(A_{0}\right)=P(A)$.

(2) If $\alpha$ and $\beta$ are any two real numbers and $(s, t) \in D$ then almost surely

$$
\left(\int(\alpha t+\beta g) d X\right)(s, t)=\alpha\left(\int f d X\right)(s, t)+\beta\left(\int g d X\right)(s, t) .
$$

(3) For every point $(s, t)$ in $D, \operatorname{var}\left[\left(\int f d X\right)(s, t)\right]=\|f\|_{s, t}$ and $E\left[\left(\int f d X\right)(s, t)\right]=0$.

Let $\chi_{[0, n]}(t)$ denote the indicator function of $[0, n]$, i.e., $\chi_{[0, n]}(t)=1$ if $0 \leqq t \leqq n$ and $\chi_{[0, n]}(t)=0$ otherwise. In order to define the stochastic integral for a function $f \in \mathfrak{M}_{2}$, we observe that $\int_{D} f^{2} d \iota \leqq n$ implies $\int_{0}^{1} \int_{0}^{t} f^{2}(u, v) d u d v \leqq n$ for all $t \in[0,1]$ and so $f(s, t, \omega)=f_{n+m}(s, t, \omega)$ for $m=0,1,2, \cdots$ where $f_{n}(s, t)=\chi_{[0, n]}\left(\int_{0}^{1} \int_{0}^{t} f^{2}(u, v) d u d v\right) f(s, t)$. Let $F_{n}=$ $\left\{\omega: \int_{D} f^{2} d \iota \leqq n\right\}$. Then $F_{n} \in \mathfrak{B}$ and $F_{1} \subset F_{2} \subset F_{3} \subset \cdots$ and from the definition of $\mathfrak{M}_{2}, P\left(\bigcup_{1}^{\infty} F_{n}\right)=1$. Using property (1) of the last theorem, we see that there is a set $F_{n, 0} \subset F_{n}$ such that $F_{n, 0} \in \mathfrak{B}, P\left(F_{n, 0}^{\prime}\right)=P\left(F_{n}\right)$ and for $\omega \in F_{n, 0},(s, t) \in D$,

$$
\left(\int f_{n} d X\right)(s, t, \omega)=\left(\int f_{n+m} d X\right)(s, t, \omega) \quad m=1,2, \cdots .
$$

Definition. Let $f$ be a function in $\mathfrak{M}_{2}$. The stochastic integral of $f$ is defined to be

$$
\left(\int f d X\right)(s, t, \omega)=\left(\int f_{n} d X\right)(s, t, \omega) \quad \text { if } \omega \in F_{n, 0}
$$

and is defined to be zero if $\omega \notin \bigcup_{n=1}^{\infty} F_{n, 0}$. 
The next theorem gives easy properties of such integrals.

THEOREM 3.3. Let $f$ and $g$ be in $\mathfrak{M}_{2}$. The following statements hold for stochastic integrals of $f$ and $g$.

(1) If $f(s, t, \omega)=g(s, t, \omega)$ on $D$ for $\omega \in A \in \mathfrak{B}$, then $\left(\int f d X\right)(s, t, \omega)=$ $\left(\int g d X\right)(s, t, \omega)$ on $D$ for $\omega \in A_{0} \in \mathfrak{B}$ where $A_{0} \subset A$ and $P\left(A_{0}\right)=P(A)$.

(2) If $\alpha$ and $\beta$ are real numbers, then

$$
\left(\int(\alpha f+\beta g) d X\right)(s, t)=\alpha\left(\int f d X\right)(s, t)+\beta\left(\int g d X\right)(s, t)
$$

holds almost surely.

4. Lemmas for Theorem 2.1. Let $K_{i j}=K((i / n),(j / n))$ where $K(s, t)$ is a real-valued continuous function on $D$ and define the transformation $T_{n}: C_{2} \rightarrow C_{2}$ by

$$
\begin{aligned}
\left(T_{n} x\right)(s, t)= & x(s, t)+\frac{1}{n^{2}} \sum_{i=1}^{[n s]} \sum_{j=1}^{[n t]} K_{i-1, j-1} x\left(\frac{i-1}{n}, \frac{j-1}{n}\right) \\
& +\frac{1}{n} \sum_{j=1}^{[n t]} K_{[n s], j-1} x\left(\frac{[n s]}{n}, \frac{j-1}{n}\right)\left(s-\frac{[n s]}{n}\right) \\
& +\frac{1}{n} \sum_{i=1}^{[n s]} K_{i-1,[n t]} x\left(\frac{i-1}{n}, \frac{[n t]}{n}\right)\left(t-\frac{[n t]}{n}\right) \\
& +K_{[n s],[n t]} x\left(\frac{[n s]}{n}, \frac{[n t]}{n}\right)\left(s-\frac{[n s]}{n}\right)\left(t-\frac{[n t]}{n}\right) .
\end{aligned}
$$

For $s=(\ell / n), t=(k / n)$, we have for $\ell, k=1,2, \cdots, n$

$$
\left(T_{n} x\right)\left(\frac{\ell}{n}, \frac{k}{n}\right)=x\left(\frac{\ell}{n}, \frac{k}{n}\right)+\frac{1}{n^{2}} \sum_{i=1}^{\ell-1} \sum_{j=1}^{k-1} K_{i j} x\left(\frac{i}{n}, \frac{j}{n}\right) .
$$

Lemma 1. Let $H\left(\eta_{11}, \cdots, \eta_{n n}\right)$ be a real-valued bounded and continuous function on $R^{n^{2}}$ and let $G(x), x \in C_{2}$, be defined by

$$
G(x)=H\left(x\left(\frac{1}{n}, \frac{1}{n}\right), \cdots, x\left(\frac{n}{n}, \frac{n}{n}\right)\right),
$$

then

$$
\begin{aligned}
\int_{C_{2}} G(x) d m= & \int_{C_{2}} G\left(T_{n} x\right) \exp \left\{-\sum_{i=1}^{n} \sum_{j=1}^{n} K_{i-1, j-1} x\left(\frac{i-1}{n}, \frac{j-1}{n} \Delta_{i j}\right\}\right. \\
& \cdot \exp \left\{-\frac{1}{2} \sum_{i=1}^{n} \sum_{j=1}^{n} K_{i-1, j-1}^{2} x^{2}\left(\frac{i-1}{n}, \frac{j-1}{n}\right) \frac{1}{n^{2}}\right\} d m
\end{aligned}
$$




$$
\Delta_{i j}=x\left(\frac{i}{n}, \frac{j}{n}\right)-x\left(\frac{i-1}{n}, \frac{j}{n}\right)-x\left(\frac{i}{n}, \frac{j-1}{n}\right)+x\left(\frac{i-1}{n}, \frac{j-1}{n}\right) .
$$

Proof. From the definition of $m$, we have

$$
\begin{aligned}
& \int_{C_{2}} G(x) d m=\left(2 \pi^{-2} n\right)^{-n^{2} / 2} \int_{R^{2}} H\left(\eta_{11}, \cdots, \eta_{m}\right) \\
& \cdot \exp \left\{-\frac{n^{2}}{2} \sum_{i=1}^{n} \sum_{j=1}^{n}\left(\eta_{i j}-\eta_{i-1, j}-\eta_{i, j-1}+\eta_{i-1, j-1}\right)^{2}\right\} d \eta_{11}, \cdots, d \eta_{n n} .
\end{aligned}
$$

Let $S_{n}$ denote the linear transformation of $R^{n^{2}}$ onto itself defined by

$$
\eta_{i j}=\xi_{i j}+\frac{1}{n^{2}} \sum_{m=1}^{i-1} \sum_{\ell=1}^{j-1} K_{m l} \xi_{m l} \quad i, j=1,2, \cdots, n .
$$

The Jacobian of $S_{n}$ is equal to 1 . Applying $S_{n}$ to the right side of (4.6) we obtain

$$
\begin{aligned}
& \int_{C 2} G(x) d m=\left(2 \pi^{-2} n\right)^{-n^{2} / 2} \\
& \cdot \int_{R^{n^{2}}} H\left(\xi_{11}, \cdots, \xi_{n n}+\frac{1}{n^{2}} \sum_{i=1}^{n-1} \sum_{j=1}^{n-1} K_{i j} \xi_{i j}\right) J_{n}\left(\xi_{11}, \cdots, \xi_{n n}\right) d \xi_{11}, \cdots, d \xi_{n n}
\end{aligned}
$$

where

$$
\begin{aligned}
& J_{n}\left(\xi_{11}, \cdots, \xi_{n n}\right) \\
& =\exp \left\{-\sum_{i=1}^{n} \sum_{j=1}^{n} K_{i-1, j-1} \xi_{i-1, j-1}\left(\xi_{i j}-\xi_{i-1, j}-\xi_{i, j-1}+\xi_{i-1, j-1}\right)\right\} \\
& \quad \cdot \exp \left\{-\frac{1}{2} \sum_{i=1}^{n} \sum_{j=1}^{n} K_{i-1, j-1}^{2} \xi_{i-1, j-1}^{2} \frac{1}{n^{2}}-\frac{n^{2}}{2} \sum_{i=1}^{n} \sum_{j=1}^{n}\left(\xi_{i j}-\xi_{i-1, j}-\xi_{i, j-1}+\xi_{i-1, j-1}\right)^{2}\right\} .
\end{aligned}
$$

On the other hand,

$$
\begin{aligned}
G\left(T_{n} x\right) & =H\left(T_{n} x\left(\frac{1}{n}, \frac{1}{n}\right), \cdots, T_{n} x\left(\frac{n}{n}, \frac{n}{n}\right)\right) \\
= & H\left(x\left(\frac{1}{n}, \frac{1}{n}\right), \cdots, x\left(\frac{n}{n}, \frac{n}{n}\right)+\frac{1}{n^{2}} \sum_{i=1}^{n-1} \sum_{j=1}^{n-1} K_{i j} x\left(\frac{i}{n}, \frac{j}{n}\right)\right) .
\end{aligned}
$$

Again from the definition of $m$, we see that the right side of (4.7) is equal to

$$
\begin{aligned}
\int_{C_{2}} G\left(T_{n} x\right) \exp \{ & -\sum_{i=1}^{n} \sum_{j=1}^{n} K_{i-1, j-1} x\left(\frac{i-1}{n}, \frac{j-1}{n}\right) \Delta_{i j} \\
& \left.-\frac{1}{2} \sum_{i=1}^{n} \sum_{j=1}^{n} K_{i-1, j-1}^{2} x^{2}\left(\frac{i-1}{n}, \frac{j-1}{n}\right) \frac{1}{n^{2}}\right\} d m
\end{aligned}
$$

which is precisely the right side of (4.5). 
LEMMA 2. Let $X$ be a random variable on a probability space $(\Omega, \mathfrak{B}, P)$ which is distributed normally with mean 0 and variance $v$. Let $Y$ be a random variable on $(\Omega, \mathfrak{B}, P)$ which is measurable with respect to a $\sigma$-algebra $\mathfrak{A} \subset \mathfrak{B}$. If the $\sigma$-algebra $\sigma\{X\} \subset \mathfrak{B}$ generated by $X$ and the $\sigma$-algebra $\mathfrak{A}$ are independent, then

$$
E\left(\exp \left\{X Y-\frac{1}{2} v Y^{2}\right\} \mid \mathfrak{I}\right)=1 \text {. }
$$

Proof. To prove the lemma we show that for every $A \in \mathfrak{A}$

$$
\int_{A} \exp \left\{X Y-\frac{1}{2} v Y^{2}\right\} d P=\int_{A} d P \text {. }
$$

Let $P_{\mathfrak{A}}$ be the restriction of $P$ to $\mathfrak{A}$. Let us write $\sigma\{\mathfrak{M}\}$ to mean the $\sigma$-algebra generated by a collection of sets $\mathfrak{M}$. Consider the transformation $T$ of the measure space $(\Omega, \mathfrak{B})$ into the measure space $\left(R^{1} \times \Omega, \sigma\left\{\mathfrak{B}^{1} \times \mathfrak{A}\right\}\right)$ defined by $T(\omega)=(X(\omega), \omega)$. This is a measurable transformation since $T^{-1}\left(\sigma\left\{\mathfrak{B}^{1} \times \mathfrak{A}\right\}\right)=\sigma\left\{T^{-1}\left(\mathfrak{B}^{1} \times \mathfrak{A}\right)\right\}$ which is contained in $\mathfrak{B}$ since $T^{-1}\left(\mathfrak{B}^{1} \times \mathfrak{U}\right)$ is. Let $U$ be the transformation of $\left(R^{1} \times \Omega, \sigma\left\{\mathfrak{B}^{1} \times \mathfrak{A}\right\}\right)$ into $\left(R^{1}, \mathfrak{S}^{1}\right)$ defined by

$$
U(\xi, \omega)=\exp \left\{\xi Y(\omega)-1 / 2 Y^{2}(\omega)\right\} .
$$

This too is a measurable transformation since $Y$ is $\mathfrak{A}$-measurable.

Let $P_{T}$ be the probability measure on $\sigma\left\{\mathfrak{B}^{1} \times \mathfrak{B}\right\}$ induced by $T$. For $B \in \mathfrak{B}^{1}$ and $A \in \mathfrak{B}$, we have from the independence of $\sigma\{X\}$ and $\mathfrak{A}$

$$
P_{T}(B \times A)=P\left(T^{-1}(B \times A)\right)=P\{X \in B\} P(A)=P_{X}(B) P \mathfrak{x}(A)
$$

where $P_{X}$ is the probability measure on $\mathfrak{B}^{1}$ induced by $X$, i.e., the normal distribution with mean 0 and variance $v$. Thus $P_{T}$ is the product measure of $P_{X}$ and $P_{\mathfrak{A}}$.

Now for $A \in \mathfrak{A}$ we have by Tonelli's Theorem

$$
\begin{aligned}
\int_{A} \exp \left\{X Y-\frac{1}{2} v Y^{2}\right\} d P & =\int_{\Omega}(U T)(\omega) d P=\int_{R^{1} \times A} U(\xi, \omega) d P_{T} \\
& =\int_{A}\left[\int_{R^{1}} \exp \left\{\xi Y(\omega)-\frac{1}{2} v Y^{2}(\omega)\right\} d P_{X}\right] d P_{\mathfrak{A}} \\
& =\int_{A}\left[\frac{1}{\sqrt{2 \pi v}} \int_{R^{1}} \exp \left\{-\frac{\left(\xi-v Y(\omega)^{2}\right.}{2 v}\right\} d \xi\right] d P_{\mathfrak{A}} \\
& =\int_{A} d P_{\mathfrak{A}} .
\end{aligned}
$$

This completes the proof of the lemma.

Lemma 3. Let $X(s, t, x)$ be the stochastic process on the probability 
space $\left(C_{2}, \mathfrak{F}, m\right)$ and the domain of definition $D=[0,1] \times[0,1]$ defined by $X(s, t, x)=x(s, t)$ for $x \in C_{2}$ and $(s, t) \in D$. Let $g(s, t)$ be a real valued function on $D$ and let $f_{n}(s, t, x)$ be a stochastic process on $\left(C_{2}, \mathfrak{F}, m\right)$ and $D$ defined by

$$
f_{n}(s, t, x)=g\left(\frac{[n s]}{n}, \frac{[n t]}{n}\right) x\left(\frac{[n s]}{n}, \frac{[n t]}{n}\right) \text { for } x \in C_{2} .
$$

Then the stochastic integral $\left(\int f_{n} d X\right)(s, t, x)$ of the process $f_{n}(s, t, x)$ with respects to the generalized Brownian motion $X(s, t, x)$ satisfies

$$
E\left[\exp \left\{\left(\int f_{n} d X\right)(1,1, x)-\frac{1}{2} \int_{D} f_{n}^{2}(s, t, x) d_{\ell}\right\}\right]=1
$$

Proof. Since $f_{n}$ is a stochastic step function,

$$
\left(\int f_{n} d X\right)(1,1)=\sum_{i=1}^{n} \sum_{j=1}^{n} f_{n}\left(\frac{i-1}{n}, \frac{j-1}{n}\right) \Delta_{i j}
$$

where

$$
\Delta_{i j}=X\left(\frac{i}{n}, \frac{j}{n}\right)-X\left(\frac{i-1}{n}, \frac{j}{n}\right)-X\left(\frac{i}{n}, \frac{j-1}{n}\right)+X\left(\frac{i-1}{n}, \frac{j-1}{n}\right) .
$$

Let

$$
T_{i j}=f_{n}\left(\frac{i-1}{n}, \frac{j-1}{n}\right) \Delta_{i j}-\frac{1}{2} f_{n}^{2}\left(\frac{i-1}{n}, \frac{j-1}{n}\right) \frac{1}{n^{2}} .
$$

Since

$$
\begin{aligned}
\int_{D} f_{n}^{2}(s, t) d \iota=\sum_{i=1}^{n} \sum_{k=1}^{n} f_{n}^{2}\left(\frac{i-1}{n}, \frac{j-1}{n}\right) \frac{1}{n^{2}}, \\
Z_{i j} \equiv \exp \left\{\int f_{n} d X\left(\frac{i}{n}, \frac{j}{n}\right)-\frac{1}{2} \int_{0}^{i / n} \int_{0}^{j / n} f_{n}^{2}(s, t) d s d t\right\} \\
=\exp \left\{\sum_{p=1}^{i} \sum_{q=1}^{j} T_{p q}\right\} .
\end{aligned}
$$

Let $\mathfrak{A}_{i j}$ denote the $\sigma$-algebra $\sigma\{X(s, t): s \leqq i / n$ or $t \leqq j / n\}$. Then $f_{n}(i-1) / n,(j-1) / n$ is $\mathfrak{A}_{i-1},{ }_{j-1}$-measurable for $i, j=1,2, \cdots, n$. The random variable $\Delta_{i j}$ is normally distributed with mean 0 and variance $1 / n^{2}$. Furthermore $\sigma\left\{\Delta_{i j}\right\}$ and $\mathfrak{A}_{i-1, j-1}$ are independent. By Lemma 2

$$
E\left(\exp \left\{T_{i j}\right\} \mid \mathfrak{I}_{i-1, j-1}\right)=1 .
$$

To prove the lemma, we must show that $E Z_{11}=1$. Now for $m=1, \cdots n X(m-1) / n, 0)=0$ and hence $\left.f_{n}(m-1) / n, 0\right)=0$ and $T_{m 1}=0$. We have $Z_{n 1}=\exp \left\{\sum_{p=1}^{n} T_{p 1}\right\}=1$. The proof will proceed by induction. 
Consider $E\left(Z_{n, j-1} \exp \left\{\sum_{m=1}^{k} T_{m j}\right\}\right)$. If $k=1$

$$
E\left(Z_{n, j-1} \exp \left\{T_{1 j}\right\}\right)=E\left[E\left(Z_{n, j-1} \exp \left\{T_{1 j}\right\} \mid \mathfrak{A}_{0, j-1}\right)\right] \text {. }
$$

Since $Z_{n, j-1}$ is $\mathfrak{2}_{0, j-1}$-measurable

$$
\begin{aligned}
E\left(Z_{n, j-1} \exp \left\{T_{1 j}\right\}\right) & =E\left[Z_{n, j-1} E\left(\exp \left\{T_{1 j}\right\} \mid \mathfrak{I}_{0, j-1}\right)\right] \\
& =E\left[Z_{n, j-1}\right]
\end{aligned}
$$

by (4.11). Now suppose that

$$
E\left(Z_{n, j-1} \exp \left\{\sum_{m=1}^{k} T_{m j}\right\}\right)=E\left(Z_{n, j-1}\right) .
$$

Then

$$
E\left(Z_{n, j-1} \exp \left\{\sum_{m=1}^{k+1} T_{m j}\right\}\right)=E\left[E\left(Z_{n, j-1} \exp \left\{\sum_{m=1}^{k+1} T_{m j}\right\} \mid \mathfrak{A}_{k, j-1}\right)\right] .
$$

Since $Z_{n, j-1} \exp \left\{\sum_{m=1}^{k} T_{m j}\right\}$ is $\mathfrak{A}_{k, j-1}$-measurable

$$
\begin{aligned}
E\left[Z_{n, j-1} \exp \left\{\sum_{m=1}^{k+1} T_{m j}\right\} \mid \mathfrak{A}_{k, j-1}\right] & =Z_{n, j-1} \exp \left\{\sum_{m=1}^{k} T_{m j}\right\} E\left(\exp \left\{T_{k+1, j}\right\} \mid \mathfrak{A}_{k, j-1}\right) \\
& =Z_{n, j-1} \exp \left\{\sum_{m=1}^{k} T_{m j}\right\} .
\end{aligned}
$$

By the induction hypothesis, we have

$$
E\left(Z_{n, j-1} \exp \left\{\sum_{m=1}^{k+1} T_{m j}\right\}\right)=E\left(Z_{n, j-1}\right) .
$$

In particular for $k=n, Z_{n j}=Z_{n, j-1} \exp \left\{\sum_{m=1}^{n} T_{m j}\right\}$ and $E Z_{n j}=E Z_{n, j-1}$. It follows that $E Z_{n n}=E_{n 1}=1$.

Let $L_{n}$ be the transformation of $C_{2}$ into $C_{2}$ defined for $(s, t) \epsilon$ $[(i-1) / n, i / n] \times[(j-1) / n, j / n]$ by

$$
\begin{aligned}
L_{n} x(s, t)= & \frac{1}{n^{2}}\left\{x\left(\frac{i}{n}, \frac{j}{n}\right)\left(s-\frac{i-1}{n}\right)\left(t-\frac{j-1}{n}\right)\right. \\
& +x\left(\frac{i-1}{n}, \frac{j}{n}\right)\left(\frac{i}{n}-s\right)\left(t-\frac{j-1}{n}\right) \\
& +x\left(\frac{i}{n}, \frac{j-1}{n}\right)\left(s-\frac{i-1}{n}\right)\left(\frac{j}{n}-t\right) \\
& \left.+x\left(\frac{i-1}{n}, \frac{j-1}{n}\right)\left(\frac{i}{n}-s\right)\left(\frac{j}{n}-t\right)\right\} .
\end{aligned}
$$

Clearly

$$
\left\|\left|L _ { n } x \left\|\left|=\max _{i, j=1, \cdots, n}\right| x\left(\frac{i}{n}, \frac{j}{n}\right)|\leqq\|x\||\right.\right.\right.
$$




$$
\lim _{n \rightarrow \infty}\left|\left\|L_{n} x-x\right\|\right| \mid=0 .
$$

Then for $T$ and $T_{n}$ defined by (1.2) and (4.1) respectively, we have

$$
\lim _{n \rightarrow \infty}\left\|L_{n} T_{n} x-T x\right\|=0 \text {. }
$$

This follows from

$$
\|\| L_{n} T_{n} x-T x\|\mid \leqq\| L_{n} T_{n} x-L_{n} T x\|+\| L_{n} T x-T x \|
$$

where

$$
\left\|L_{n} T_{n} x-L_{n} T x\right\| \mid \leqq\left\|T_{n} x-T x\right\| \| \text { by }(4.13), \lim _{n \rightarrow \infty}\left\|T_{n} x-T x\right\|=0
$$

from the uniform continuity of $K(s, t)$ on $D$, and $\lim _{n \rightarrow \infty}\|\| L_{n} T x-T x\|\|=0$ by (4.14).

Lemma 4. Let $X(s, t, x), g(s, t)$ and $f_{n}(s, t, x)$ be as defined in Lemma 3 . Then the random variables $Z_{n}(x), n=1,2, \cdots$, on $\left(C_{2}, \mathfrak{F}, m\right)$ defined by

$$
Z_{n}=\exp \left\{\left(\int f_{n} d X\right)(1,1)-\int_{D} f_{n}^{2}(s, t) d \iota\right\}
$$

are uniformly integrable on $C_{2}$. If $g(s, t)$ is bounded on $D$, then for every $B \geqq 0$, the random variables $Y_{n}(x), n=1,2, \cdots$, defined by

$$
Y_{n}(x)=\chi_{[0, B]}\left(\|\| L_{n} x \|||\right) \exp \left\{\left(\int f_{n} d X\right)(1,1)\right\}
$$

are uniformly integrable on $C_{2}$.

Proof. For $M>0$ let $A_{M, n}=\left\{x \in C_{2}: Z_{n}(x)>M\right\}$. To show the uniform integrability of $Z_{n}, n=1,2, \cdots$, we show that for every $\varepsilon>0$ there exists $M>0$ independent of $n$ such that

$$
\int_{A_{M, n}} Z_{n}(x) d m<\varepsilon \quad n=1,2, \cdots .
$$

According to Lemma 3 applied to $2 f_{n}, E\left(Z_{n}^{2}\right)=1$ and so choosing $M>1 / \varepsilon$, we have

$$
\int_{A_{M, n}} Z_{n}(x) d m \leqq \int_{A_{M, n}} \frac{1}{M} Z_{n}^{2}(x) d m \leqq \frac{1}{M}<\varepsilon
$$

proving the uniform integrability of $Z_{n}, n=1,2, \cdots$.

Suppose $g(s, t)$ is bounded on $D$. Now

$$
\text { || } L_{n} x\left|\|=\max _{(s, t) \in D}\right| X\left(\frac{[n s]}{n}, \frac{[n t]}{n}, x\right) \mid
$$


and so if $x \in C_{2},\left\|\mid L_{n} x\right\| \leqq B$, and $B>0$, then $\left\|\left|f_{n}\|\mid \leqq B\| g\|\|\right.\right.$ and $\int_{D} f_{n}^{2}(s, t) d_{\ell} \leqq B^{2}\left\||g \||^{2}\right.$. Letting $\gamma=\exp \left\{\|\mid\| \|^{2} B^{2}\right\}$ we have

$$
Y_{n}(x)=\chi_{[0, B]}\left(\left\|L_{n} x\right\|\right) Z_{n}(x) \exp \left\{\int_{D} f_{n}^{2}(s, t) d \ell\right\} \leqq \gamma Z_{n}(x) .
$$

For $K>\gamma \varepsilon^{-1}$

$$
\int_{\left[Y_{n}>K\right]} Y_{n}(x) d m \leqq \gamma \int_{\left[Z_{n}>K / r\right]} Z_{n}(x) d m<\varepsilon \gamma \quad n=1,2, \cdots
$$

proving the uniform integrability of $Y_{n}, n=1,2, \cdots$.

Lemma 5. If $x \in C_{2}$ and for some $M>0\left\|L_{n} x\right\| \|>M \exp \{\mid\|K\|\}$, then $\left\|\left|L_{n} T_{n} x \|\right|>M\right.$.

Proof. As in the Volterra integral theory one can show that $T_{n}$ defined by (4.1) transforms $C_{2}$ onto $C_{2}$ in a one-to-one manner, $T_{n}$ and $T_{n}^{-1}$ are bounded linear operators, and $\left\|T_{n}^{-1}\right\| \leqq \exp \{\||| K\| \mid\}$.

Now for any $x \in C_{2}$ which satisfies $\||x|\|>M \exp \{\|K\| \mid \|\}$ for some $M>0$, we have $\|x\|>M\left\|T_{n}^{-1}\right\|$. If $\left\|T_{n} x\right\| \leqq M$,

$$
M\left\|T_{n}^{-1}\right\|<\|\| x\||=|\| T_{n}^{-1} T_{n} x\left\|\left|\leqq\left\|T_{n}^{-1}\right\| \cdot\left\|T_{n} x\right\|\right| \leqq\right\| T_{n}^{-1} \| M
$$

a contradiction. Thus for any $x \in C_{2}$, if $\|x\|>M\left\|T_{n}^{-1}\right\|$ then II $T_{n} x\|\|>M$. In particular $\left\|L_{n} x\right\| \mid>M \exp \{\||| K \mid\|\}$ implies $\left\|T_{n} L_{n} x\right\| \mid>M$. But (4.1) and (4.12) imply that $T_{n} L_{n} x=L_{n} T_{n} x$ and hence $\left\|L_{n} T_{n} x\right\| \mid>M$.

5. Proof of Theorem 1. Since $L_{n} x, x \in C_{2}$, is determined by the values of $x$ on the lattice points $(i / n, j / n) i, j=1,2, \cdots, n$, we may define a function $H$ on $R^{n^{2}}$ by

$$
H\left(\eta_{11}, \cdots, \eta_{n n}\right)=F\left(L_{n} x\right)
$$

where $\eta_{i j}=x(i / n, j / n)$. The continuity of $F$ and $L_{n}$ implies the $\mathfrak{F}$ measurability of $F \circ L_{n}$. If $R^{n^{2}}$ is topologized according to the supnorm, it is easy to see that $H$ is continuous. Since $F$ is bounded, so is $H$. Let

$$
G(y)=F\left(L_{n} y\right)=H\left(y\left(\frac{1}{n}, \frac{1}{n}\right), \cdots, y\left(\frac{n}{n}, \frac{n}{n}\right)\right) .
$$

Then $G\left(T_{n} y\right)=F\left(L_{n} T_{n} y\right)$ and according to Lemma 1

$$
\begin{aligned}
& \int_{C_{2}} F\left(L_{n} x\right) d m=\int_{C_{2}} F\left(L_{n} T_{n} x\right) \\
& \quad \exp \left\{-\sum_{i=1}^{n} \sum_{j=1}^{n} K\left(\frac{i-1}{n}, \frac{j-1}{n}\right) x\left(\frac{i-1}{n}, \frac{j-1}{n}\right) \Delta_{i j}\right\} \\
& \quad \exp \left\{-\frac{1}{2} \sum_{i=1}^{n} \sum_{j=1}^{n} K^{2}\left(\frac{i-1}{n}, \frac{j-1}{n}\right) x^{2}\left(\frac{i-1}{n}, \frac{j-1}{n}\right) \frac{1}{n^{2}}\right\} d m .
\end{aligned}
$$


Since $\lim _{n \rightarrow \infty}||\left|L_{n} x-x\right|||=0$ and $F$ is continuous, $\lim _{n \rightarrow \infty} F\left(L_{n} x\right)=F(x)$. Since $F$ is bounded,

$$
\lim _{n \rightarrow \infty} \int_{C_{2}} F\left(L_{n} x\right) d m=\int_{C_{2}} F(x) d m .
$$

We now show that the integral on the right side of 5.1 converges to

$$
\int_{C_{2}} F(T x) \exp \left\{-\left(\int K(s, t) X(s, t) d X\right)(1,1)-\frac{1}{2} \int_{D} K^{2}(s, t) x^{2}(s, t) d_{\ell}\right\} d m
$$

which will complete the proof. Since $F$ vanishes off a bounded set, there exists $M>0$ such that if $\|x\| \mid>M$ then $F(x)=0$. Let $N=$ $M \exp \{\|K\| \mid\}$. Then

$$
\int_{C_{2}} F\left(L_{n} T_{n} x\right) J_{n}(x) d m=\int_{C_{2}} \chi_{[0, N]}\left(||\left|L_{n} x\right|||\right) F\left(L_{n} T_{n} x\right) J_{n}(x) d m
$$

where

$$
\begin{aligned}
J_{n}(x)= & \exp \left\{-\sum_{i=1}^{n} \sum_{j=1}^{n} K\left(\frac{i-1}{n}, \frac{j-1}{n}\right) x\left(\frac{i-1}{n}, \frac{j-1}{n}\right) \Delta_{i j}\right\} \\
& \cdot \exp \left\{-\frac{1}{2} \sum_{i=1}^{n} \sum_{j=1}^{n} K^{2}\left(\frac{i-1}{n}, \frac{j-1}{n}\right) x^{2}\left(\frac{i-1}{n}, \frac{j-1}{n}\right) \frac{1}{n^{2}}\right\} .
\end{aligned}
$$

Since $K(s, t)$ and $x(s, t)$ are continuous on $D$

$$
\begin{aligned}
\lim _{n \rightarrow \infty} \exp \{ & \left.-\frac{1}{2}-\sum_{i=1}^{n} \sum_{j=1}^{n} K^{2}\left(\frac{i-1}{n}, \frac{j-1}{n}\right) x^{2}\left(\frac{i-1}{n}, \frac{j-1}{n}\right) \frac{1}{n^{2}}\right\} \\
= & \exp \left\{-\frac{1}{2} \int_{D} K^{2}(s, t) x^{2}(s, t) d \iota\right\} .
\end{aligned}
$$

Let

$$
f_{n}(s, t)=K\left(\frac{[n s]}{n}, \frac{[n t]}{n}\right) X\left(\frac{[n s]}{n}, \frac{[n t]}{n}\right)
$$

and $f(s, t)=K(s, t) X(s, t)$. Then

$$
\sum_{i=1}^{n} \sum_{j=1}^{n} K\left(\frac{i-1}{n}, \frac{j-1}{n}\right) x\left(\frac{i-1}{n}, \frac{j-1}{n}\right) \Delta_{i j}=\left(\int f_{n} d X\right)(1,1, x) .
$$

By Theorem 3.2, since $f_{n} \in \mathfrak{M}_{1}$ and $f \in \mathfrak{M}_{1}$

$$
\begin{aligned}
\operatorname{var}\left[\left(\int f_{n} d X\right)(1,1, x)-\left(\int f d X\right)(1,1, x)\right] & =\operatorname{var}\left[\left(\int\left(f_{n}-f\right) d X\right)(1,1, x)\right] \\
& \leqq \int_{D} E\left(f_{n}-f\right)^{2} d_{\iota}=\left\|f_{n}-f_{n}\right\|^{2} .
\end{aligned}
$$

Now 


$$
\begin{aligned}
\left\|f-f_{n}\right\| \leqq & \|X(s, t)\| \cdot\left\|K(s, t)-K\left(\frac{[n s]}{n}, \frac{[n t]}{n}\right)\right\| \| \\
& +\|\| K\|\cdot\|\left\|X(s, t)-X\left(\frac{[n s]}{n}, \frac{[n t]}{n}\right)\right\| \| .
\end{aligned}
$$

Using the continuity of $X(s, t)$ and $K(s, t)$ on $D$ and the fact that $X(s, t)-X([n s] / n,[n t] / n)$ is normally distributed with mean 0 and variance $s t-([n s] / n) \cdot([n t] / n)$ it is easy to see that $\lim _{n \rightarrow \infty}\left\|f_{n}-f\right\|=0$. It follows that $\left(\int f_{n} d X\right)(1,1, x)$ converges in $m$-measure to $\left(\int f d X\right)(1,1, x)$ and hence

$$
\exp \left\{-\sum_{i=1}^{n} \sum_{j=1}^{n} K\left(\frac{i-1}{n}, \frac{j-1}{n}\right) x\left(\frac{i-1}{n}, \frac{j-1}{n}\right) \Delta_{i j}\right\}
$$

converges in $m$-measure to $\exp \left\{-\left(\int K(s, t) x(s, t) d X\right)(1,1)\right\}$.

The integrand on the right side of (5.3) converges in $m$-measure to

$$
\begin{aligned}
\chi_{[0, N]}(\||| x|| \mid) F(T x) \exp \{ & -\left(\int K(s, t) X(s, t) d X\right)(1,1) \\
& \left.-\frac{1}{2} \int_{D} K^{2}(s, t) x^{2}(s, t) d \iota\right\} .
\end{aligned}
$$

This follows from the above and the fact that $\left\|L_{n} x\right\|\|\leqq\| x \|$ and $\lim _{n \rightarrow \infty}\|\| L_{n} x-x\|\|=0$, which implies

$$
\lim _{n \rightarrow \infty} \chi_{\ulcorner 0, N]}\left(||\left|L_{n} x \|\right| \mid\right)=\chi_{[0, N]}(|\|x\|| \mid) \text {. }
$$

Since for each $n=1,2, \cdots$, the integrands on the right side of (5.3) are bounded in absolute value by

$$
\chi_{[0, N]}\left(\left\||| L_{n} x \mid\right\|\right)\||| F \mid\| \exp \left\{-\left(\int f_{n} d X\right)(1,1)\right\} .
$$

Lemma 4 implies that the integrands are uniformly integrable justifying the taking of limits inside the integral. Thus

$$
\begin{aligned}
\lim _{n \rightarrow \infty} \int_{C_{2}} F\left(L_{n} T_{n} x\right) J_{n}(x) d m & =\lim _{n \rightarrow \infty} \int_{C_{2}} \chi_{[0, N]}\left(\left\||| L_{n} x\right\|||\right) F\left(L_{n} T_{n} x\right) J_{n}(x) d m \\
& =\int_{C_{2}} \chi_{[0, N]}(\|x|\|| \mid) F(T x) J(x) d m
\end{aligned}
$$

where

$$
J(x)=\exp \left\{-\left(\int K(s, t) X(s, t) d X\right)(1,1)-\frac{1}{2} \int_{D} K^{2}(s, t) x^{2}(s, t) d \ell\right\} .
$$

Now $\|x\| \| N$ implies $\|T x\| \|>M$ and hence $F(T x)=0$. We get finally 


$$
\lim _{n \rightarrow \infty} \int_{C_{2}} F\left(L_{n} T_{n} x\right) J_{n}(x) d m=\int_{C_{2}} F(T x) J(x) d m
$$

which upon substitution into (5.1) proves Theorem 1.

The proof of Theorem 2 is proved using Theorem 1 in exactly the same way as Theorem III is proved from Theorem II in [4] by J. Yeh and is therefore omitted.

The author wishes to thank Professor J. Yeh for suggesting this problem.

\section{REFERENCES}

1. R. H. Cameron and W.T. Martin, Transformations of Wiener integrals under a general class of linear transformations, Trans. Amer. Math. Soc. 58 (1945), 184-219. 2. K. Ito, On the stochastic differential equations, Memoirs Amer. Math. Soc. 4 (1951). 3. J. Yeh, Wiener measure in a space of functions of two variables, Trans. Amer. Math. Soc. 95 (1960), 433-450.

4. Cameron-Martin translation theorems in the Wiener space of functions of two variables, Trans. Amer. Math. Soc. 107 (1963), 409-420.

Received April 21, 1970. The paper is part of the author's thesis. The research was supported in part by the National Science Foundation (Grant No. GP-13288) and in part by the Air Force (Grant No. 1321-67).

University of California, Santa Barbara 



\section{PACIFIC JOURNAL OF MATHEMATICS}

\section{EDITORS}

H. SAMELson

Stanford University

Stanford, California 94305

R. R. PHelPS

University of Washington

Seattle, Washington 98105
J. Dugundu

Department of Mathematics

University of Southern Californle

Los Angeles, California 9.0007

RICHARD ARENS

University of California

Los Angeles, California 9.0024

\section{ASSOCIATE EDITORS}
E. F. BECKENBACH
B. H. NeumanN
F. WOLE
K. Yoshida

\section{SUPPORTING INSTITUTIONS}

UNIVERSITY OF BRITISH COLUMBIA

CALIFORNIA INSTITUTE OF TECHNOLOGY

UNIVERSITY OF CALIFORNIA

MONTANA STATE UNIVERSITY

UNIVERSITY OF NEVADA

NEW MEXICO STATE UNIVERSITY

OREGON STATE UNIVERSITY

UNIVERSITY OF OREGON

OSAKA UNIVERSITY

UNIVERSITY OF SOUTHERN CALIFORNIA

\author{
STANFORD UNIVERSITY \\ UNIVERSITY OF TOKYO \\ UNIVERSITY OF UTAH \\ WASHINGTON STATE UNIVERSITY \\ UNIVERSITY OF WASHINGTON \\ AMERICAN MATHEMATICAL SOCIETY \\ CHEVRON RESEARCH CORPORATION \\ NAVAL WEAPONS CENTER
}

The Supporting Institutions listed above contribute to the cost of publication of this Journal, but they are not owners or publishers and have no responsibility for its content or policies.

Mathematical papers intended for publication in the Pacific Journal of Mathematics should be in typed form or offset-reproduced, (not dittoed), double spaced with large margins. Underline Greek letters in red, German in green, and script in blue. The first paragraph or two must be capable of being used separately as a synopsis of the entire paper. The editorial "we" must not be used in the synopsis, and items of the bibliography should not be cited there unless absolutely necessary, in which case they must be identified by author and Journal, rather than by item number. Manuscripts, in duplicate if possible, may be sent to any one of the four editors. Please classify according to the scheme of Math. Rev. Index to Vol. 39. All other communications to the editors should be addressed to the managing editor, Richard Arens, University of California, Los Angeles, California, 90024.

50 reprints are provided free for each article; additional copies may be obtained at cost in multiples of 50 .

The Pacific Journal of Mathematics is published monthly. Effective with Volume 16 the price per volume (3 numbers) is $\$ 8.00$; single issues, $\$ 3.00$. Special price for current issues to individual faculty members of supporting institutions and to individual members of the American Mathematical Society: $\$ 4.00$ per volume; single issues $\$ 1.50$. Back numbers are available.

Subscriptions, orders for back numbers, and changes of address should be sent to Pacific Journal of Mathematics, 103 Highland Boulevard, Berkeley, California, 94708.

PUBLISHED BY PACIFIC JOURNAL OF MATHEMATICS, A NON-PROFIT CORPORATION

Printed at Kokusai Bunken Insatsusha (International Academic Printing Co., Ltd.), 7-17, Fujimi 2-chome, Chiyoda-ku, Tokyo, Japan. 


\section{Pacific Journal of Mathematics}

\section{Vol. 36, No. 2 December, 1971}

George E. Andrews, On a partition problem of H. L. Alder ............ 279

Thomas Craig Brown, An interesting combinatorial method in the theory of locally finite semigroups .......................... 285

Yuen-Kwok Chan, A constructive proof of Sard's theorem ............. 291

Charles Vernon Coffman, Spectral theory of monotone Hammerstein

operators...................................... 303

Edward Dewey Davis, Regular sequences and minimal bases .......... 323

Israel (Yitzchak) Nathan Herstein and Lance W. Small, Regular elements in

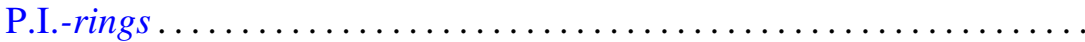

Marcel Herzog, Intersections of nilpotent Hall subgroups ..............

W. N. Hudson, Volterra transformations of the Wiener measure on the space

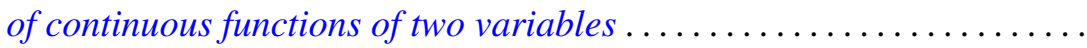

J. H. V. Hunt, An n-arc theorem for Peano spaces ................ 351

Arnold Joseph Insel, A decomposition theorem for topological group

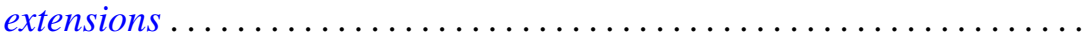

Caulton Lee Irwin, Inverting operators for singular boundary value

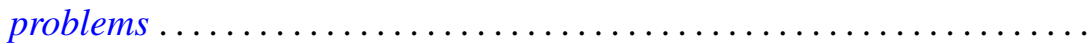

Abraham A. Klein, Matrix rings of finite degree of nilpotency ............ 387

Wei-Eihn Kuan, On the hyperplane section through a rational point of an algebraic variety...

John Hathway Lindsey, II, On a six-dimensional projective representation of $\mathrm{PSU}_{4}(3)$

Jorge Martinez, Approximation by archimedean lattice cones ...

J. F. McClendon, On stable fiber space obstructions .........

Mitsuru Nakai and Leo Sario, Behavior of Green lines at the Kuramochi boundary of a Riemann surface ....................

Donald Steven Passman, Linear identities in group rings. I. .

Donald Steven Passman, Linear identities in group rings. II ...

David S. Promislow, The Kakutani theorem for tensor products of

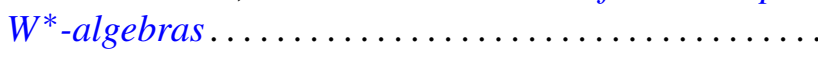

Richard Lewis Roth, On the conjugating representation of a finite group

Bert Alan Taylor, On weighted polynomial approximation of entire functions...

William Charles Waterhouse, Divisor classes in pseudo Galois

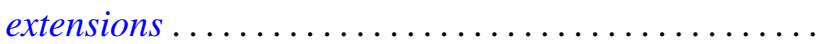

Chi Song Wong, Subadditive functions ...

Ta-Sun $\mathrm{Wu}$, A note on the minimality of certain bitransformation groups 